\title{
Confinement in Coulomb gauge
}

\section{G. Burgio*}

Institut für Theoretische Physik

Auf der Morgenstelle 14

72076 Tübingen

Germany

E-mail: giuseppe.burgio@uni-tuebingen. de

\section{Markus Quandt}

Institut für Theoretische Physik

Auf der Morgenstelle 14

72076 Tübingen

Germany

E-mail: markus.quandteuni-tuebingen. de

\section{Hugo Reinhardt}

Institut für Theoretische Physik

Auf der Morgenstelle 14

72076 Tübingen

Germany

E-mail: hugo.reinhardteuni-tuebingen. de

\section{Mario Schröck}

Institut für Physik, FB Theoretische Physik

Universität Graz

8010 Graz

Austria

E-mail: mario.schroeckeuni-graz.at

\section{H. Vogt}

Institut für Theoretische Physik

Auf der Morgenstelle 14

72076 Tübingen

Germany

E-mail: hannes.vogt@uni-tuebingen. de

We review our lattice results concerning the Gribov-Zwanziger confinement mechanism in Coulomb gauge. In particular, we verify the validity of Gribov's IR divergence condition for the Coulomb ghost form factor. We also show how the quark self energy is, like that of the transverse gluon, IR divergent, thus effectively extending the Gribov-Zwanziger scenario to full QCD.

31st International Symposium on Lattice Field Theory - LATTICE 2013

July 29 - August 3, 2013

Mainz, Germany

\footnotetext{
* Speaker.
} 


\section{Introduction}

Gribov was the first to notice [1] that for non-Abelian theories most gauge conditions admit several solutions and the corresponding Faddeev-Popov (FP) mechanism is not sufficient to define the functional integral beyond perturbation theory. The field-configuration space must therefore be restricted to a domain, continuously connected to the origin, where the gauge condition possesses unique solutions. He then showed how, as soon as the fields cross the boundary of such region, the ghost dressing function acquires a singularity; his "no-pole" condition for the FP-ghost at nonvanishing momentum is then necessary to implement the restriction to the so called Gribov region.

In particular, in Coulomb gauge, he argued how such restriction can imply a diverging gluon self-energy, motivating its disappearance from the physical spectrum. QCD in Coulomb gauge has since then been a fruitful playground in investigating the Gribov-Zwanziger (GZ) confinement mechanism [1,2]. In a series of papers $[3,4,5,6,7,8]$, briefly summarized here, we have analyzed the behaviour of the relevant two-point functions at zero temperature on the lattice and compared them with the corresponding predictions of Hamiltonian variational calculations [9, 10, 11].

Gribov based his conjectures on more or less heuristic arguments, which Zwanziger later tried to put on a more solid basis, while variational calculations, viable in Coulomb gauge since they by-pass the explicit construction of the gauge invariant Hilbert space [12], did provide some insight on the relation of the GZ-mechanism to the Hamiltonian formulation. In both cases, however, approximations need to be made; although many authors tackled the problems during the years $[13,14,15,16,17,18]$, a satisfactory non-perturbative cross-check from lattice calculation was hindered for a long time by the presence of strong discretization effects. We have shown $[3,7,8]$ how for each propagator a mixture of improved actions and separate treatment of their energy dependence can quite effectively solve such problems, allowing an explicit check of the GZ-scenario. In particular, anisotropic actions prove to be very useful [19, 20]; details can be found in [8], as well as a description of the gauge fixing algorithm, which adapts those introduced in [22, 23]. Following the ideas in [3], a first anisotropic analysis in SU(3) had been attempted in [24].

From the continuum analysis and from our results in [3,4] we know that in the pure gauge sector the static gluon propagator, the static Coulomb potential and the ghost form factor should obey:

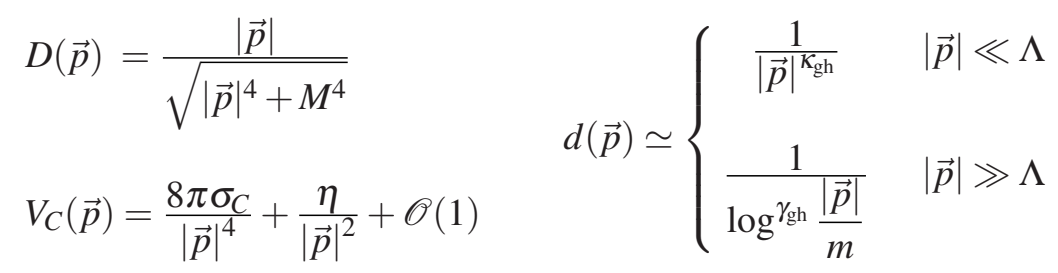

where the Gribov mass $M \simeq 1 \mathrm{GeV}$ and for the gluon self-energy $\omega_{A}=D^{-1}(\vec{p})$ holds. The quark propagator, the fermion self energy and the running mass $M(|\vec{p}|)$ should take the form [7]:

$$
\begin{gathered}
S\left(\vec{p}, p_{4}\right)=\frac{Z(\vec{p})}{i \vec{p}+i p_{4} \alpha(\vec{p})+M(\vec{p})} \quad \omega_{F}(|\vec{p}|)=\frac{\alpha(|\vec{p}|)}{Z^{n}(|\vec{p}|)} \sqrt{\vec{p}^{2}+M^{2}(|\vec{p}|)} \\
M(|\vec{p}|)=\frac{m_{\chi}\left(m_{b}\right)}{1+b \frac{|\vec{p}|^{2}}{\Lambda^{2}} \log \left(e+\frac{|\vec{p}|^{2}}{\Lambda^{2}}\right)^{-\gamma}}+\frac{m_{r}\left(m_{b}\right)}{\log \left(e+\frac{|\vec{p}|^{2}}{\Lambda^{2}}\right)^{\gamma}}
\end{gathered}
$$


where $Z$ is the field renormalization function, $\alpha$ the energy renormalization function, $m_{b}$ the bare quark mass, $m_{\chi}\left(m_{b}\right)$ the chiral mass and $m_{r}\left(m_{b}\right)$ the renormalized running mass [7]. The exponent $n$ in the rhs of $\omega_{F}$ depends on the exact definition of the self energy to be compared with the hamiltonian approach.

\section{Results}

\subsection{Ghost form factor}

A careful analysis of the ghost form factor in the Hamiltonian limit $a_{t} \rightarrow 0$ shows that its UV behaviour agrees with Eq. (1.1), with $\gamma_{\mathrm{gh}}=1 / 2$, confirming continuum predictions, and $m=$ $0.21(1) \mathrm{GeV}$, see Fig. 1 (a). In the IR going to higher anisotropies increases the exponent $\kappa_{\mathrm{gh}}$, as shown in Fig. 1 (b), where we plot $|\vec{p}|^{\kappa_{m}} d(\vec{p})$, with $\kappa_{m}$ the IR exponent for $\xi=1$, as a function of the anisotropy. The limit $\xi \rightarrow \infty$ gives $\kappa_{\text {gh }} \gtrsim 0.5$, confirming the GZ-scenario. This however

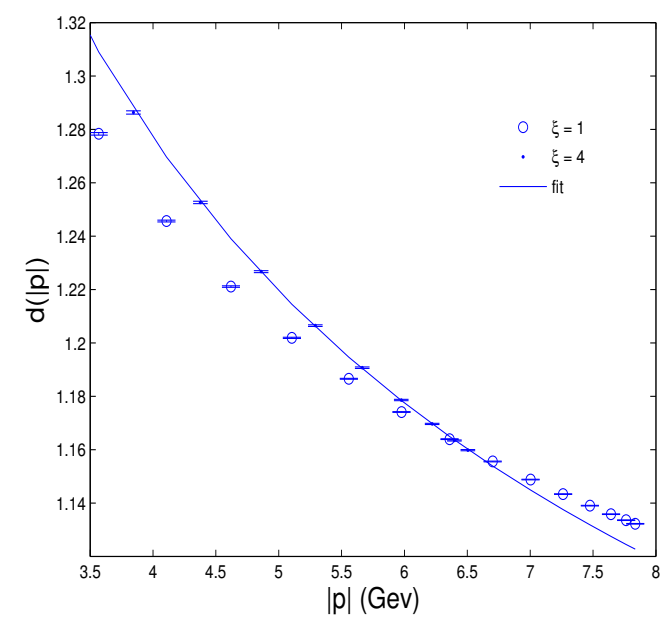

(a)

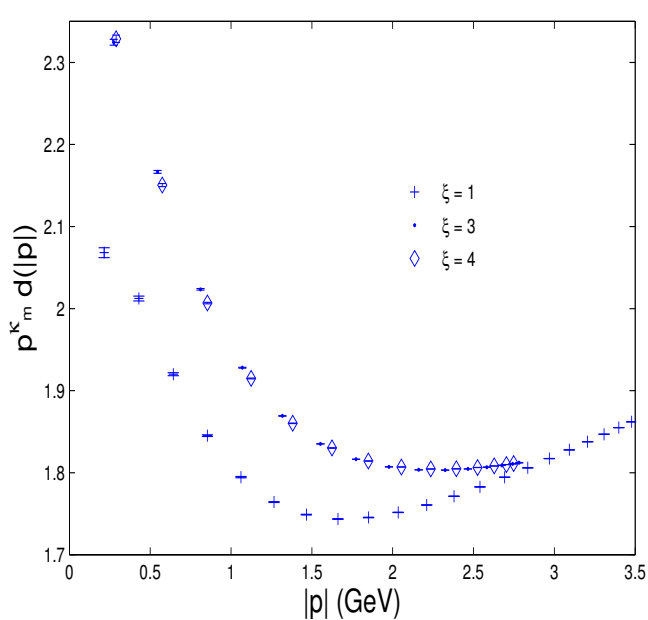

(b)

Figure 1: (a): UV behavior of $d(\vec{p})$ compared with Eq. (1.1). (b): IR behavior of $|\vec{p}|^{\kappa_{m}} d(\vec{p})$, both for different anisotropies $\xi$.

disagrees with some continuum predictions $\kappa_{\mathrm{gh}}=1$, deriving from the assumption of the finiteness of the static ghost-gluon vertex. Whether this is indeed correct and algorithmic improvements could change the lattice result is still a matter of investigation.

\subsection{Coulomb potential}

In Fig. 2 (a) we show $|\vec{p}|^{4} V_{C}(|\vec{p}|)$ as obtained from different anisotropies. Somewhat boldly fitting the results to Eq. (1.1) we get, in the Hamiltonian limit $\xi \rightarrow \infty, \sigma_{C}=2.2(2) \sigma$ as expected from Zwanziger's predictions [25]. See the talk of H. Vogt in this conference for a more "honest" discussion. 


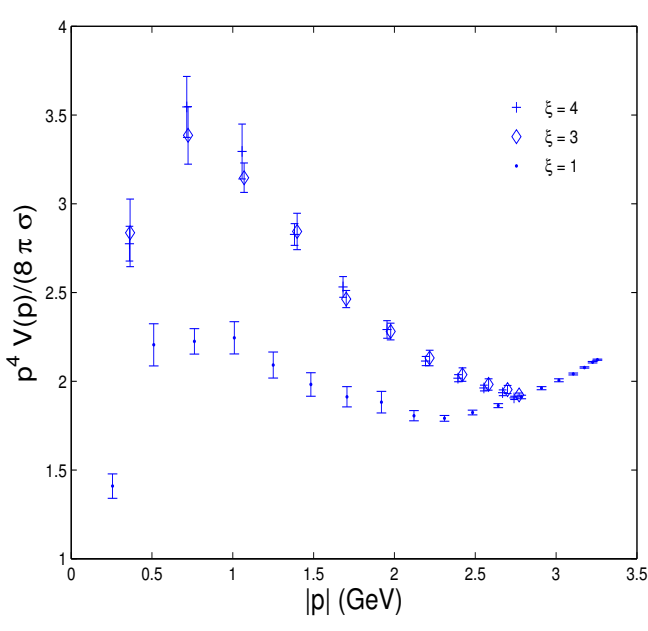

(a)

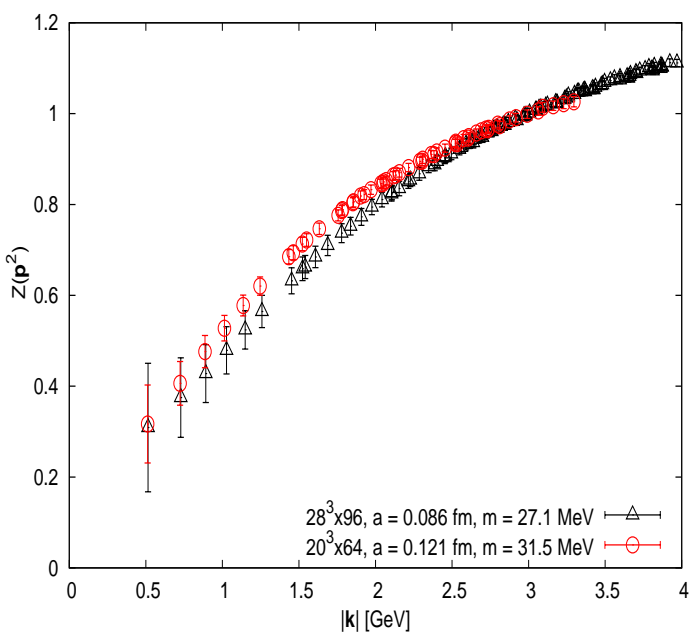

(b)

Figure 2: (a): Infrared behavior of $|\vec{p}|^{4} V_{C}(\vec{p}) /(8 \pi \sigma)$ for different anisotropies $\xi$. (b): Quark field renormalization function $Z(|\vec{p}|)$.

\subsection{Quark propagator}

Our calculations were all made on a set of configurations generated by the MILC collaboration [26], see [7] for details. The use of improved actions is crucial to establish the scaling properties of the Coulomb gauge quark propagators. This is very similar to the situation in Landau gauge, see e.g. $[27,28,29]$, whose techniques we have adapted to our case.

Fig. 2 (b) shows the scaling of the renormalization function $Z(|\vec{p}|)$ for configurations calculated at similar bare quark mass, while the RG-invariant functions $\alpha(|\vec{p}|)$ and $M(|\vec{p}|)$ are given in Fig. 3. Their behaviour agrees with theoretical expectations, see Eq. (1.2).

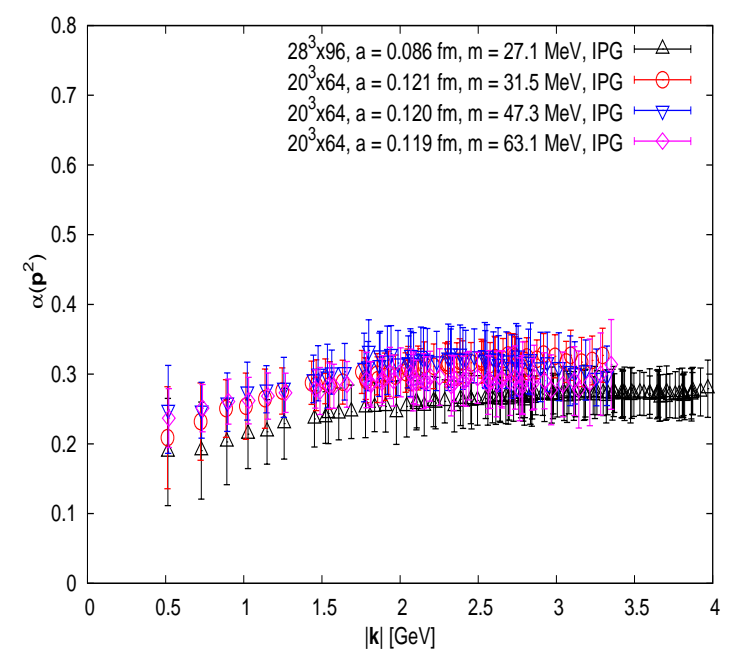

(a)

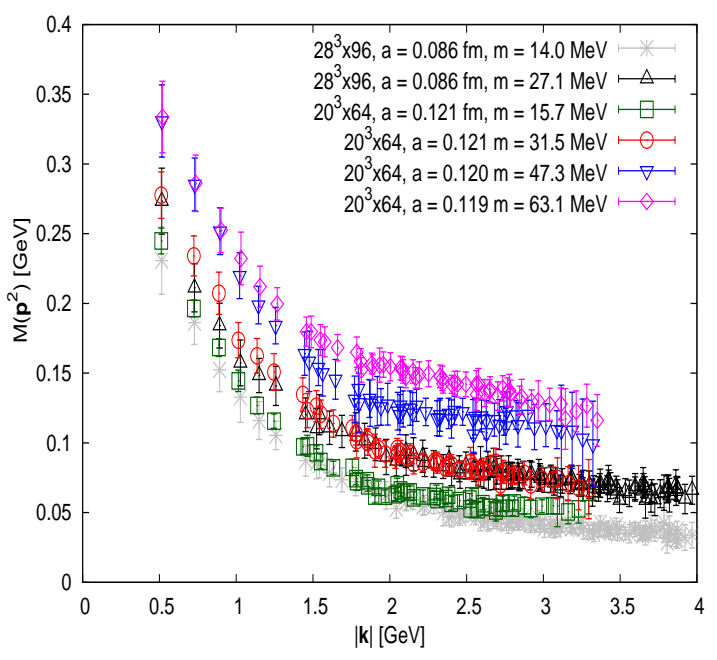

(b)

Figure 3: (a): Energy renormalization function $\alpha(|\vec{p}|)$. (b): Running mass $M(|\vec{p}|)$. 
Our most interesting results are given in Fig. 4. Analogously to the gluon self-energy $\omega_{A}(|\vec{p}|)$, the quark self energy $\omega_{F}(|\vec{p}|)$ has a turn-over at $|\vec{p}| \sim 1 \mathrm{GeV}$, clearly departing from the behaviour of a free particle, and diverging in the IR, see Fig. 4 (a); although awaiting confirmation on larger lattices, this extends the Gribov argument to full QCD. Moreover, as Fig. 4 (b) shows, the running mass $M(|\vec{p}|)$ we obtain is quantitatively compatible with our phenomenological expectations from chiral symmetry breaking. Fitting it to Eq. (1.2) we obtain $b=2.9(1), \gamma=0.84(2)$, $\Lambda=1.22(6) \mathrm{GeV}, m_{\chi}(0)=0.31(1) \mathrm{GeV}$, with $\chi^{2} /$ d.o.f. $=1.06$.

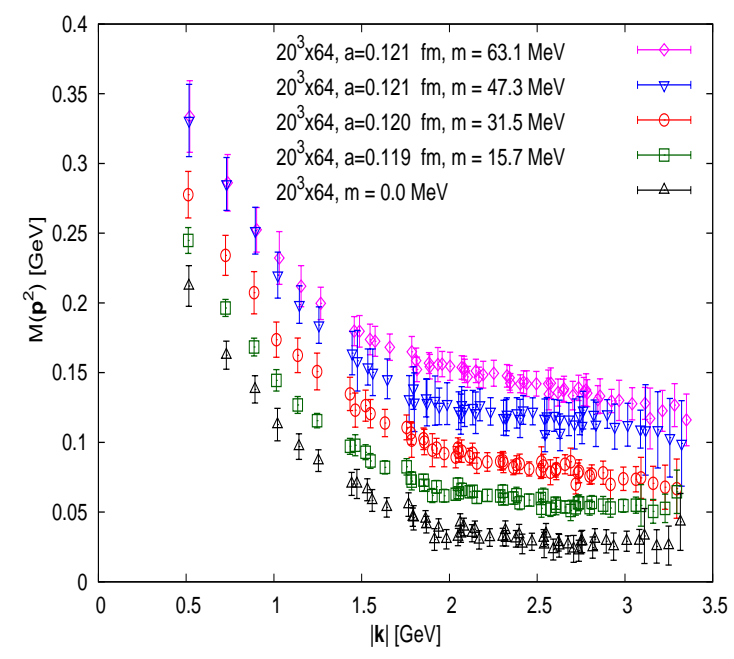

(a)

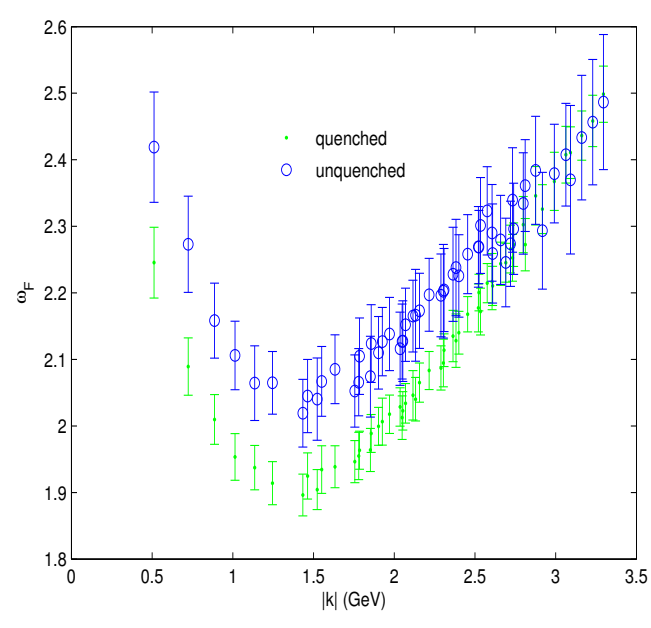

(b)

Figure 4: (a): Quark self energy $\omega_{F}(|\vec{p}|)$. (b): Running mass $M(|\vec{p}|)$ in the chiral limit $m_{b} \rightarrow 0$; see Eq. (1.2).

\section{Conclusions}

We have shown that lattice calculations confirm the GZ confinement scenario in Coulomb gauge at $T=0$. The ghost form factor $d(|\vec{p}|)$ is IR divergent with an exponent $\kappa_{\mathrm{gh}} \gtrsim 0.5$, which implies Gribov's no-pole condition and a dual-superconducting scenario [30]; the gluon propagator satisfies the Gribov formula, implying an IR diverging self-energy, and the Coulomb potential seems compatible with a string tension roughly twice the physical string tension. Moreover from the quark propagator we can extract the quark self energy $\omega_{F}(|\vec{p}|)$, which is compatible with an IR divergent behaviour, and the running mass $M(|\vec{p}|)$, which gives a constituent quark mass of $m_{\chi}(0)=0.31(1) \mathrm{GeV}$.

The situation in Coulomb gauge seem to be easier than in Landau gauge, where BRST symmetry is non-perturbatively broken, violating the Kugo-Ojima confinement scenario [31], while the GZ confinement scenario is realized explicitly introducing of an horizon function, see e.g. [32] for a recent review; its physical implications and how these can be related to the presence of dim-2 condensates [33, 34, 35] are an interesting issue still debated in the literature [36].

\section{Aknowledgements}

This work was partially supprted the DFG under the contract DFG-Re856/6-3. 


\section{References}

[1] V. N. Gribov, "Quantization of non-Abelian gauge theories," Nucl. Phys., vol. B139, p. 1, 1978.

[2] D. Zwanziger, "Lattice Coulomb Hamiltonian and static color-Coulomb field," Nucl. Phys., vol. B485, pp. 185-240, 1997.

[3] G. Burgio, M. Quandt, and H. Reinhardt, "Coulomb gauge gluon propagator and the Gribov formula," Phys. Rev. Lett., vol. 102, p. 032002, 2009.

[4] G. Burgio, M. Quandt, and H. Reinhardt, "BRST symmetry vs. Horizon condition in Yang-Mills theories," Phys. Rev., vol. D81, p. 074502, 2010.

[5] M. Quandt, H. Reinhardt, and G. Burgio, “The role of center vortices in Gribov's confinement scenario,” Phys. Rev., vol. D81, p. 065016, 2010.

[6] H. Reinhardt, M. Quandt, and G. Burgio, "Temporal wilson loop in the hamiltonian approach in coulomb gauge,” Phys. Rev. D, vol. 85, p. 025001, 2012.

[7] G. Burgio, M. Schrock, H. Reinhardt, and M. Quandt, "Running mass, effective energy and confinement: the lattice quark propagator in Coulomb gauge," Phys.Rev., vol. D86, p. 014506, 2012.

[8] G. Burgio, M. Quandt, and H. Reinhardt, "Ghost propagator and the Coulomb form factor from the lattice," Phys.Rev., vol. D86, p. 045029, 2012.

[9] A. P. Szczepaniak and E. S. Swanson, "Coulomb gauge QCD, confinement, and the constituent representation,” Phys. Rev., vol. D65, p. 025012, 2002.

[10] C. Feuchter and H. Reinhardt, "Variational solution of the Yang-Mills Schroedinger equation in Coulomb gauge,” Phys. Rev., vol. D70, p. 105021, 2004.

[11] D. Epple, H. Reinhardt, and W. Schleifenbaum, "Confining Solution of the Dyson-Schwinger Equations in Coulomb Gauge," Phys. Rev., vol. D75, p. 045011, 2007.

[12] G. Burgio, R. De Pietri, H. A. Morales-Tecotl, L. F. Urrutia, and J. D. Vergara, "The basis of the physical Hilbert space of lattice gauge theories," Nucl. Phys., vol. B566, pp. 547-561, 2000.

[13] A. Cucchieri and D. Zwanziger, "Fit to gluon propagator and Gribov formula," Phys. Lett., vol. B524, pp. 123-128, 2002.

[14] K. Langfeld and L. Moyaerts, "Propagators in Coulomb gauge from SU(2) lattice gauge theory," Phys. Rev., vol. D70, p. 074507, 2004.

[15] Y. Nakagawa, A. Nakamura, T. Saito, and H. Toki, "Infrared behavior of the Faddeev-Popov operator in Coulomb gauge QCD,” Phys.Rev., vol. D75, p. 014508, 2007.

[16] A. Voigt, E.-M. Ilgenfritz, M. Muller-Preussker, and A. Sternbeck, "The Effective Coulomb potential in SU(3) lattice Yang-Mills theory," Phys.Rev., vol. D78, p. 014501, 2008.

[17] Y. Nakagawa, A. Nakamura, T. Saito, and T. Toki, “The Coulomb gauge confinement scenario and the color-dependent quark potentials in lattice QCD simulations," Mod.Phys.Lett., vol. A23, pp. 2348-2351, 2008.

[18] Y. Nakagawa, A. Nakamura, T. Saito, and H. Toki, "Confining time-like gluon and confined spatial gluons in Coulomb gauge QCD,” Mod.Phys.Lett., vol. A23, pp. 2352-2355, 2008.

[19] G. Burgio, S. Caracciolo, and A. Pelissetto, "Algebraic algorithm for the computation of one loop Feynman diagrams in lattice QCD with Wilson fermions,” Nucl.Phys., vol. B478, pp. 687-722, 1996. 
[20] G. Burgio, A. Feo, M. J. Peardon, and S. M. Ryan, "Gauge Theories on a 2+2 Anisotropic Lattice," Phys. Rev., vol. D67, p. 114502, 2003.

[21] K. Ishiguro, T. Suzuki, and T. Yazawa, "Effective monopole action at finite temperature in SU(2) gluodynamics," JHEP, vol. 0201, p. 038, 2002.

[22] I. L. Bogolubsky et al., "Landau gauge ghost and gluon propagators in SU(2) lattice gauge theory: Gribov ambiguity revisited," Phys. Rev., vol. D74, p. 034503, 2006.

[23] I. L. Bogolubsky et al., "Improved Landau gauge fixing and the suppression of finite-volume effects of the lattice gluon propagator," Phys. Rev., vol. D77, p. 014504, 2008.

[24] Y. Nakagawa, A. Nakamura, T. Saito, and H. Toki, "Scaling study of the gluon propagator in Coulomb gauge QCD on isotropic and anisotropic lattices,” Phys.Rev., vol. D83, p. 114503, 2011.

[25] D. Zwanziger, "No confinement without Coulomb confinement," Phys.Rev.Lett., vol. 90, p. 102001, 2003.

[26] A. Bazavov, D. Toussaint, C. Bernard, J. Laiho, C. DeTar, et al., "Nonperturbative QCD simulations with 2+1 flavors of improved staggered quarks," Rev.Mod.Phys., vol. 82, pp. 1349-1417, 2010.

[27] P. O. Bowman, U. M. Heller, and A. G. Williams, "Lattice quark propagator with staggered quarks in Landau and Laplacian gauges,” Phys.Rev., vol. D66, p. 014505, 2002.

[28] P. O. Bowman, U. M. Heller, D. B. Leinweber, M. B. Parappilly, A. G. Williams, et al., "Unquenched quark propagator in Landau gauge," Phys.Rev., vol. D71, p. 054507, 2005.

[29] M. B. Parappilly et al., "Scaling behavior of quark propagator in full QCD," Phys.Rev., vol. D73, p. $054504,2006$.

[30] H. Reinhardt, “The dielectric function of the QCD vacuum,” Phys. Rev. Lett., vol. 101, p. 061602, 2008.

[31] T. Kugo and I. Ojima, "Local Covariant Operator Formalism of Nonabelian Gauge Theories and Quark Confinement Problem,” Prog. Theor. Phys. Suppl., vol. 66, p. 1, 1979.

[32] N. Vandersickel and D. Zwanziger, “The Gribov problem and QCD dynamics," Phys.Rept., vol. 520, pp. 175-251, 2012.

[33] G. Burgio, F. Di Renzo, G. Marchesini, and E. Onofri, " $\Lambda^{2}$-contribution to the condensate in lattice gauge theory," Phys. Lett., vol. B422, pp. 219-226, 1998.

[34] R. Akhoury and V. I. Zakharov, "On non perturbative corrections to the potential for heavy quarks," Phys. Lett., vol. B438, pp. 165-172, 1998.

[35] P. Boucaud et al., "Lattice calculation of $\frac{1}{p^{2}}$ corrections to $\alpha(s)$ and of $\Lambda_{Q C D}$ in the $\widetilde{M O M}$ scheme," JHEP, vol. 04, p. 006, 2000.

[36] A. Cucchieri, D. Dudal, T. Mendes, and N. Vandersickel, "Modeling the Gluon Propagator in Landau Gauge: Lattice Estimates of Pole Masses and Dimension-Two Condensates," Phys.Rev., vol. D85, p. 094513, 2012. 